\title{
Stellar Populations Across cD Galaxies
}

\author{
Susan I. Loubser ${ }^{1}$, A.E. Sansom ${ }^{1}$ \\ and I.K. Soechting ${ }^{2}$ \\ ${ }^{1}$ Centre for Astrophysics, University of Central Lancashire, Preston, PR1 2HE, UK \\ email: siloubser@uclan.ac.uk \\ ${ }^{2}$ Oxford Astrophysics, Department of Physics, University of Oxford, \\ Oxford, OX1 3RH, UK
}

\begin{abstract}
We present preliminary long-slit spectroscopy of a sample of nearby $\mathrm{cD}$ galaxies recently observed with the William Herschel and GEMINI North and South Telescopes. So far, we have accumulated data for $27 \mathrm{cDs}$. More is being observed with the GEMINI telescopes, to create a statistically significant sample. Unresolved stellar populations will be used to investigate the history of when stars formed in these galaxies. Central and spatially resolved line strengths measured from these high signal-to-noise data will be interpreted via population modelling and used to derive luminosity weighted average ages and abundances. Comparison with previous results from other Hubble types of galaxies will highlight relative differences in their evolution.
\end{abstract}

Keywords. galaxies: elliptical and lenticular, cD, galaxies: formation, galaxies: stellar content

\section{Introduction}

cD galaxies are amongst the largest and brightest in the universe, and occur in the centres of rich clusters. They are embedded in an extensive luminous halo. They are similar to elliptical galaxies, but their formation history is expected to differ due to the unusual location and extreme luminosity of cDs. This project is aimed at determining when the stars formed in $\mathrm{cD}$ galaxies. New spectroscopic observations, together with the use of existing galactic chemical evolution models will be used to derive stellar population ages and star formation histories in $\mathrm{cD}$ galaxies.

The formation, characteristics and locations of $\mathrm{cD}$ galaxies in galaxy clusters are well modeled by mergers of galaxies, referred to in the literature as "galactic cannibalism" (e.g. Jordán et al. 2004). Observations of multiple nuclei in central galaxies favours this theory. It is possible at higher redshift to see the progenitors of $\mathrm{cD}$ galaxies as multiple cluster galaxies come together. Another theory proposes that $\mathrm{cD}$ galaxy halos are formed by cooling flows in clusters of galaxies. If the central cluster density is high enough, intracluster gas can condense and form stars at the bottom of the potential well. However, this possibility now seems remote in light of XMM-Newton observations that show that the X-ray gas does not cool significantly (Jordán et al. 2004). cD galaxies also contain unexpected amounts of line emitting and molecular gas compared to elliptical galaxies (Bettoni, Galletta \& Garćia-Burillo 2003). This indicate that ongoing star formation may be occurring, whereas other theories suggest that the galaxy mergers are mainly "dry mergers" unaccompanied by gas and star formation.

\section{Galaxy Sample and Observations}

We selected a large sample of $\mathrm{cD}$ galaxies satisfying the folowing criteria: classified as a cD galaxy in the NASA Extragalactic Database; bright apparent magnitude $m_{B}<15.5$; in the local universe (distance $<320 \mathrm{Mpc}$ ); and not in the galactic plane $\left(\left|b_{2}\right|>15^{\circ}\right)$. 


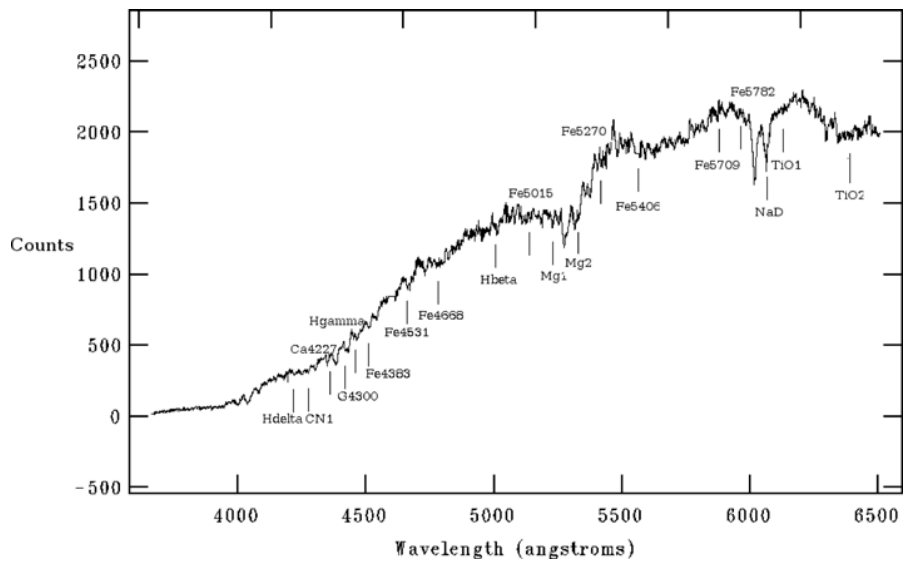

Figure 1. Gemini GMOS-South spectrum of the central 0.9 arcseconds of the cD galaxy NGC7012. This shows that we can achieve a S/N ratio of 30 per $\AA$ over a broad wavelength range in the centres of $\mathrm{cD}$ galaxies with one-hour exposures.

We also applied an absolute magnitude cutoff at $M_{B}=-20$ to avoid misclassified, lower luminosity galaxies. Four different selection methods were used to select the final sample: the Bautz-Morgan and Rood-Sastry galaxy cluster classification systems; all-sky search in HyperLeda for galaxies of T-type $=-4$; published surface brightness profiles in literature; and X-ray studies of brightest cluster galaxies.

A subset of ten of the brightest galaxies selected were observed on the $4.2 \mathrm{~m}$ WHT telescope in June 2006. Further to this we are obtaining observations on the Gemini North and South telescopes. Our aim is to acquire a total sample of $\sim 50 \mathrm{cD}$ galaxies to measure the spread of star formation behaviours and ages amongst the $\mathrm{cD}$ class.

\section{Modeling Absorption Lines}

With spectroscopic optical absorption line strengths, covering a broad spectral range, we can measure average luminosity-weighted ages and metallicities to within $\sim 20 \%$. Our self-consistent galactic chemical evolution code (Sansom \& Proctor 1998; Proctor \& Sansom 2002) can model the chemical evolution of stellar populations in galaxies. This code uses models of luminosity-weighted populations of stars (Thomas, Maraston \& Korn 2004) to predict spectral features on the Lick/IDS system. Observed absorption line indices will be fit to model predictions using $\chi^{2}$ (Proctor \& Sansom 2002). Galactic cannibalism could set up a number of star formation episodes, leading to an extended duration of star formation. On the other hand, cooling flows may result in an unusual mass distribution (dominated by lower mass stars) indicated by a separate stellar population characterizing the halos of cDs. Old, short duration star formation episodes in all cD galaxies would support the picture of dry mergers. Thus, these observations will add to our understanding of how giant spheriodal galaxies of different types form.

\section{References}

Jordán, A., Côte, P., West, M.J., Marzke, R.O., Minniti, D. \& Rejkuba, M. 2004, AJ 127, 24

Bettoni, D., Galletta, G. \& García-Burillo, S. 2003, A\&A 405, 5

Proctor, R.N. \& Sansom, A.E. 2002, MNRAS 333, 517

Sansom, A.E. \& Proctor, R.N. 1998, MNRAS 297, 953

Thomas, D., Maraston, C. \& Korn, A. 2004, MNRAS 351, 19 